\title{
Ein Beitrag zu Versuchen über postmortale Pigmentbildung.
}

\author{
Von \\ Prof. Dr. Rudolf Winternitz, \\ Leiter der Abteilung für Dermat. u. Syph. an der deutschen Universitätspoliklinik \\ in Prag.
}

In überlebenden Organen, Herz, Leber, Nieren u. a. ist unter entsprechenden Bedingungen durch eine gewisse Zeit eine Fortdauer einzelner normaler mechanischer und chemischer Funktionen $\mathrm{zu}$ beobachten. Hiedurch lassen sich die Bildungsstätten gewisser Sekrete und Stoffwechselprodukte sowie verschiedene Entstehungsbedingungen letzterer sicherstellen. Für die Haut ist schon seit längerer Zeit, durch anatomische Untersuchungen (Retterer, Jarisch, ${ }^{\text {) }}$ Garcia, F. Winkler, H. Rab l) namentlich jene beim Farbenwechsel winterwei(jer Tiere (Schwalbe $)^{2}$ ), durch verschiedene Überlegungen (Kaposi) ${ }^{3}$ ), endlich durch Experimente mit künstlicher Belichtung (Grund, Meirowsky) ${ }^{4}$ ) eine in der Epidermis selbst vor sich gehende Pigmentbildung sehr wahrscheinlich geworden.

1) Jarisch. Über Anat. u. Entw. d. Oberhautp. b. Frosche. A. D. S. 1891, XXIII, p. 559. Die Arbeiten v. Retterer, Garcia, F. Winkler u. H. Rabl s. bei Meirowsky: Urspr. d. melan. Pigm. ete. Leipzig 1908.

a) Schwalbe, G. Ü. d. Farbenwechsel winterweißer Tiere etc. Morph. Arb. 1893, II., $3 \mathrm{H}$.

s) Ka posi. Ü. Pathog. d. Pigm. u. Entf. d. Haut. A. D. S. 1891, XXIII, p. 192.

4) M eirowsky. Ü. d. Pigmentierungsvorg. otc. Monatsh. f. pr. D. 1907, B. 44 (S. ebenda: Bd. 42 u. 43 ). 
Die jüngsten, sehr bemerkenswerten Untersuchungen von Meirowsky ${ }^{1}$ ) und Königstein ${ }^{2}$ ) scheinen nun die endgültige Entscheidung über den Ort der Pigmentbildung gebracht zu haben, indem sie zeigten, dal auch in (von Lebenden oder von der Leiche) exstirpierter Haut bei höherer Umgebungstemperatur und entsprechender Feuchtigkeit Pigment in sehr bedeutender Menge auftreten könne. Wenn man Haut vom Lebenden und nach dem Tode - bis ungefähr $1 \frac{1}{2}$ Tage nach letzterem (M e ir ow s k y) - mit physiologischer Kochsalzlösung befeuchtet, oder in mit dieser getränkten Gazestreifen eingebunden, in geschlossenen Eprouvetten $1-3$ Tage im Thermostat $\left(37^{\circ} \mathrm{C}\right)$ oder Paraffinofen $\left(58^{\circ} \mathrm{C}\right)$ hält, beobachte man eine gelb- bis schwarzbraune Verfärbung der Epidermis der betreffenden Hautstückchen.

Diese Verfärbung bleibt in manchen Fällen aus; so in Haut über einem Mammakarzinom (Meirowsky) oder nächst einem Gelenkfungus. Die mikroskopische Untersuchung ergab beiden Autoren Vermehrung des Pigments u. zw. nur des in der Epidermis befindlichen (Meirowsky) oder auch jenes im Korium (Königstein). In der Epidermis war Pigment in der Basalschichte - aber auch in höheren Zellagen vorhanden, also ähnlich wie in physiologisch stärker pigmentierten Stellen der Menschenund Tierhant. Doch wird auf einen Gegensatz zwischen makro- und mikroskopischem Befund hingewiesen, indem der tief dunklen Färbung der erwärmten Hautstücke keine gleich große Vermehrung des Pigmentes im mikroskopischen Bilde entspricht.

Me i r o w sk y bringt das Auftreten postmortaler Pigmentvermebrung mit Lebensvorgängen in Zusammenhang, obzwar die Zellen selbst, in welehen das Pigment entsteht, nicht mebr leben müssen. Die betreffenden Vorgänge sieht er als fermentative an. Es sei ihm gelungen, „in tierischer und menschlicher Haut eine Oxydase nachzuweisen, die auf Adrenalin, Suprarenin und Epirenan eine stark oxydierende Wirkung ausübt".

Auf Grund sehr gründlicher und sinnreicher Versuche kommt aber Königstein zu Resultaten, die den Grad der postmortalen Pigmentierung einschränken und statt der Fermenthypothese Meirowskys eine andere chemische Erklärung setzen. Er nimmt an, daB durch verschiedene experimentelle Eingriffe ein Lipoid, welches das Zusammenfließen von "Pigmentquellen" in den Zellen hindert, beseitigt wird und dab so der vermehrte Farbstoff entsteht.

1) Meirowsky. Über Pigmentbildung in vom Körper losgelöster Haut. Frankf. Zeitschr. f. Path. II 4 u. Über d. Urspr. d. melan. Pigm. d. Haut u. d. Auges, Leipzig 1908.

2) Königstein. Über postmortale Pigmentbildung. Münch. m. Woch. 1909, Nr. 45 u. Wien. kl. Woch. 1910, Nr. 17: Über d. Bez. gesteigerter Pigmentbildung z. d. Nebennieren. 
Ein Beitrag zu Versuchen über postmortale Pigmentbildung. 295

Meine Versuche, die ich größtenteils noch vor Königsteins Publikation angestellt hatte, bestätigen die Angaben beider Autoren bezüglich der objektiven Daten; zu einer Erklärung der postmortalen und vitalen Pigmentierung der Haut konnte ich aber auf Grund von Experimenten nicht kommen. Von meinen zahlreichen Versuchen will ich nur solche anführen, die meinen Schlüssen, welche den von Meirowsky und $\mathrm{K} \ddot{\mathrm{n}} \mathrm{ig}$ ste in gezogenen ähnlich sind, zur Grundlage dienen.

Ich habe von Operierten und Leichen exstirpierte Hautstücke direkt oder nach einer Vorbehandlung in Salzsäure, Ameisensäure, Wasserstoffsuperoxydlösung oder in Wasserstoffstrom bei $37^{\circ}$ und $58^{\circ}$ erwärmt, habe an postmortal pigmentierten Hautstücken Entfärbung mit Salz-, Ameisensäure oder Wasserstoffsuperoxyd vorgenommen und die entfärbten Hautstücke einer abermaligen Erwärmung ausgesetzt. Durch Einwirkung reduzierender Substanzen (Adrenalin-, Hydrochinonlösungen) auf exstirpierte Hautstücke wurde auf Vorhandensein freien Sauerstoffs geprüft; endlich versuchte ich einen oxydierresp. pigmentierbaren Stoff (Andrenalin) in der Haut nachzuweisen.

\section{Erwärmungsversuche.}

Die Methodik derselben glich jener bei Meirowsky und Königstein. Die exstirpierten Hautstücke warden in mit physiologischer Kochsalzlösung getränkten Gazestreifen eingebunden in verschlossenen Eprouvetten verwabrt, oder auf den mit genannter Lösung befeuchteten Boden von Glasdosen gelegt. Einige Tropfen Toluol, der Kochsalzlösung zugesetzt, verhüteten die Fäulnis. In mehreren Experimenten wurden (nach Zelloidin-, Paraffineinbettung oder Gefrierung) mikroskopische Schnitte angefertigt und nngefärbt untersucht.

a) Folgender Versuch, einer von vielen, wiederholt die Beobachtung der genannten Autoren.

Von der frischen Haut eines amputierten Schenkels kommt

Stück $A$ in Zimmertemperatur,

$B$ in den Brutofen (bei $37^{\circ}$ ),

bis $75^{\circ} \mathrm{C}$ stieg).

" C in den Paraffinofen $\left(58^{\circ}\right.$, dessen Temperatur diesmal

Nach einem Tag ist $B$ deutlich, $C$ sehr dunkel; beide Stücke, namentlich $C$, geschrumpft und trocken. 
Die Paraffinschnitte von $B$ sind in der Basalschicht der Epidermis dunkler als die von $A$; die $C$-Schnitte sind sowohl in der Basalschichte als böher hinauf stärker pigmentiert. Die Schnittdicken sind aber nicht gleich, da die stark geschrumpften, eingetrockneten und daher brüchigeren Stücke $B$ und $C$ die Gewinnung gleich dünner Schnitte wie $A$ nicht gestatten. Zelloidinschnitte, die von allen Stücken ungefähr gleich dick erhalten werden, ergeben viel geringere Unterschiede, namentlich ist eine Pigmentierung höherer Zellschichten nicht vorhanden.

Resumé dieses und mehrerer ähnlicher Versuche: Die in der Wärme gehaltenen Stücke sind deutlich geschrumpft, im mikroskopischen Bilde erscheinen die Retezapfen sehr verschmächtigt und die Gewebe in jeder Richtung aneinandergedrängt.

b) Die folgendeu Versuche bestätigen die Angabe ron Meirowski bezüglich mangelhaften Nachdunkelns ron Haut Karzinomatöser; sie zeigen weiters, daß stärkere Feuchtigkeit das Nachdunkeln und die Schrumpfung hindert und daß durch 3 Tage feucht gehaltene Stücke, die nicht mehr als überlebend bezeichnet werden können, durch nachheriges Eintrocknen und Erwärmen noch recht nachzudunkeln vermögen.

Versuch: Achselhaut einer 60jähr. Frau (Mammakarzinom). Zwei Stunden nach der Exstirpation werden die Stücke $A_{1}, B_{1}, C_{1}$ in Zimmertemperatur, $37^{\circ}, 51^{\circ}$; die Stücke $A_{2}, B_{2}, C_{2}$ in Kohlensäureatmosphäre wohl verschlossen, in dieselben Temperaturen gebracht.

Nach 1 bzw. 2 Tagen ist nur Stück $C_{1}\left(51^{\circ} \mathrm{C}\right)$ etwas bräunlicher pigmentiert, als die übrigen Stücke, aber auch deutlich geschrumpft, dagegen sind $A_{2}, B_{2}, C_{2}$ (sämtlich wohl verschlossen in $\mathrm{CO}_{2}$ ) nicht geschrumpft, aber etwas grauer als die Kontrollstücke - wohl durch Autolyse.

Ebenso sinnfällig bezüglich Wichtigkeit der Austrocknung und bezüglich der langdauernden Fähigkeit der Haut nachzudunkeln verlief folgender Versuch:

Mammabaut einer 25j. Frau (Carcin. mam.), 7 Stunden nach der Exstirpation. 3 Doppelproben, je eine $A_{2}, B_{2}, C_{2}$ in Kohlensäureatmosphäre, alle stark durchfeuchtet, werden bei Zimmertemperatur, $37.5^{\circ}$ und $51^{\circ}$, gehalten.

Nach 16 Stunden keine Veränderung.

$\eta 40 \quad$ erscheint nur $C_{1}$ eine Spur bräunlicher.

"64" " " $C_{1} ", "$

Nun werden bei $B_{1}, C_{1}$ die Stöpsel nur lose geschlossen, so daß Austrocknung erfolgen kann.

Nach weiteren 2 Tagen ist nur $C_{1}$ bräunlich. 
Ein Beitrag zu Versuchen über postmortale Pigmentbildung. 297

Nun kamen sämtliche Stücke $A_{1}$ und ${ }_{2}, B_{1}$ und ${ }_{2}, C_{1}$ und ${ }_{2}$ bei halbgelüfteten Stöpseln auf 2 Tage in $54^{\circ} \mathrm{C}$.

Es wird :

$A_{1}$ ziemlich trocken, kleiner als 2 Tage vorher und leicht graubräunlich;

$A_{2}$ vollständig feucht, nicht geschrumpft, eine Spur schmutziggrau (Autolyse);

$B_{1}$ etwas feucht, eine Spur brauner aussehend als vor 2 Tagen;

$B_{2}$ fast trocken, eine Spur brauner als vor 2 Tagen;

$C_{1}$ trocken, stark geschrumpft, braun;

$C_{2}$ noch etwas feucht, deutlich geschrumpft, an den Rändern eine Spur bräunlich.

Auch im nächsten Versuch - Rückenhaut eines gesunden 22jähr. Mannes - ergibt sich, daß die durch 3 Tage in mit Kochsalz getränkten Gazelappen bei Zimmertemperatur gehaltenen Hautstückchen nicht schrumpfen und weiB bleiben, hierauf aber bej $57-58^{\circ}$ gehaltenen schrumpfen, nach 24 Stunden dunkelbraun, nach weiteren 24 Stunden weiter geschrumpft und dunkelgraubraun geworden sind. Noch länger ist das Intervall 2 wischen Tod und Erwärmung in einem Versuch, wo zuerst drei, später sieben Tage nach dem (Verbrennungs-) Tode von einer Leiche Hautstückchen entnommen und in $57-58^{\circ} \mathrm{C}$ gebracht wurden. Neben der brãunlichen war bei diesem letzten Intervall auch schon schwärzlich graue Verfärbung (Fäulnis?) vorhanden.

c) Einfache Austrocknung im Exsikkatorgibt gleichfalls eine dunklere Verfärbung, die aber durch nachheriges Quellen der Hautstücke in Wasser oder stark verdünntem Alkohol gröBtenteils schwindet; dagegen hellt sich die durch Erwärmen im Paraffinofen entstandene postmortale Verfärbung durch Quellen our zu einem weit geringeren Teile auf, aber auch die Schrumpfung selber ist nicht mehr erheblich auszugleichen.

1. Versuch. Ein Fibroma pendulum vom oberen Augenlid eines brünetten jungen Mannes mit rötlicher, an zwei hanfkorngroßen Stellen dunkel pigmentierter Oberfläche wird in zwei Hälften geteilt; die eine $A$ verbleibt bei Zimmertemperatur, die andere $B$ im Exsikkator. $B$ ist nach 1 bzw. 2 Tagen in jeder Richtung geschrumpft - Messung mit Zirkelspitzen -; die Epidermis ist schwarzbraun, das früher weiße Bindegewebe gelb wie Horn geworden. $B$ wird hierauf 1 Tag in $48 \%$ igen, am nächsten Tag in 32\%igen, am dritten in $16 \%$ igen Alkohol gebracht; es quillt auf, die tiefdunkle Epidermisfarbe übergeht in eine graue, die aber doch dunkler erscheint als jene von $A$.

2. Versu ch. In zwei Stückchen von der Rückenhaut eines gesunden 22 jähr Mannes (behufs Narbenplastik exzidiert) werden in den Exsikkator $(A)$ und in den Parafinofen $(B)$ bei $58^{\circ}-59^{\circ}$ gebracht. 
$A$ ist nach 1 Tage an den Rändern eine Spur gebräunt, nach 2 Tagen weiter geschrumpft, die Oberfläche bräunlich, das ganze Stück hornartig durchscheinend, im Wasser quillt es und zeigt nach mehreren Stunden eine grauere Oberfläche als ein Kontrollstück, das Bindegewebe wird wieder weiB.

$B$ ist nach 1 Tag gleichfalls geschrumpft, schön braun wie eine blassere Negerhaut, hierauf einen Tag in $48 \%$ igen Alkohol, einen Tag in Wasser gegeben, quillt es kaum auf, bleibt graubräunlich, wenn auch etwas heller als nach der Paraffinofenerwärmung (Kontrollstück).

Resumé: Schrumpfung und postmortale Pigmentzunahme gehen ziemlich parallel (Königstein). Schon im Brutofen $\left(37^{\circ}\right)$ findet eine deutliche Verkleinerung der exstirpierten Hautstïckchen statt, beim Verweilen im Paraffinofen können die Seitenlängen des Stückes (Länge und Breite) um die Hälfte, die Dicke desselben ebenfalls beträchtlich abnehmen, was somit einer Volumsverkleinerung auf $1 / 4$ bis $1 / 8$ des Ursprünglichen entspricht.

Es muß somit bei Erhaltenbleiben des normalen Hautpigments im überlebendem Stiuck die Färbung desselben nach Verweilen im Paraffinofen 4 bis $8 \mathrm{mal}$ erhöht erscheinen, auch wenn keine Neubildung von Pigment stattgefunden hätte. W i e die makroskopische ist aber a ch diemikroskopische Pigmentierung wohl nur zu einem kleineren Teil einer wirklichen Pigmentneubildung zuzuschreiben. Die Schrumpfung nähert eben alle pigmentführenden Gebilde der Epidermis, verkleinert auch die Konturen der Pigmentzellen und rerdichtet somit das Pigment selber. Durch die Schrumpfung der Stücke gelangen aber auch basale und etwas höhere Epithel-Zellagen, welche de norma in verschiedener Höhe über der Papillenbasis liegen und sonst in verschiedene mikroskopische Schnitte kommen, in dieselben Schnittllächen, besonders auch deshalb, weil die Schnitte der höher temperierten Hautstücke namentlich nach Zelloidin- und Paraffineinbettung dicker sind als jene von den Kontrollstücken.

Hiedurch allein muß schon der Eindruck entstehen, daß in den erwärmten Stücken auch höhere Zellagen über der Basalschicht stärker pigmentiert erscheinen als de norma.

Wenn trotzdem, wie schon Meirowski und Königstein angeben, der makroskopisch sehr dunkeln Färbung der Stücke keine ebenso 
Ein Beitrag zu Versuchen über postmortale Pigmentbildung. 299

auffallende mikroskopische Pigmentvermehrung entspricht, so liegt dies zum Teile wenigstens für Zelloidin- und Paraffineinbettung daran, daß auch in den Kontrollstücken durch diese Einbettungsmethoden Sehrumpfung und Pigmentverdichtung eintritt, welche aber allerdings jene durch die vorangehende tagelange Erwärmung der Versuchsstücke nicht erreicht. Andernteils ist es aber nicht unmöglich, daß ein Teil des bei Erwärmung - Röstung - entstehenden Hautpigmentes durch verschiedene Flässigkeiten - Alkohol, Äther, Wasser - gelöst wird, welche bei der Einbettung und Traeparation verwendet werden.

Es ist nach dem Gesagten der Grad einer durch postmortales Erwärmen der Haut (bei $37^{\circ}-58^{\circ}$ ) stattindenden Pigmentneubildung für beträchtlich geringer zu taxieren, als der Augenschein annebmen läßt. Die Existenz dieser letzteren ist aber trotzdem sicher; denn erstens läßt sich durch einfaches Eintrocknen keine gleichwertige nicht reversible Färbung hervorrufen, zweitens lehrt ihr Ausbleiben in manchen Fällen - Karzinom, alter Leichenhaut - daßB in den positiven Fällen ein durch Wärme sich färbender Zellbestandteil vorhanden sein müsse.

\section{II.}

Dieser Bildungsstoff des Pigmentes besitzt innerhalb gewisser Grenzen eine größere Resistenz gegenüber biotischen und chemischen Vorgängen.

Deshalb ist in überlebender Haut nicht nur längere Zeit bis fast zum Beginn der Fäulnis die Fähigkeit der Haut, bei Erwärmung dunkel zu werden, vorhanden; auch einige chemische Operationen zerstören diese Eigenschaft nicht, falls die Reagentien nicht eine bestimmte Konzentration übersteigen.

Versuch: Stückchen normaler Haut werden 1 Tag in äquimolekularen Lösungen von Salzsäure ( $1 / 10$ Normal) resp. Wasserstoffsuperoxyd (1.13 $\mathrm{H}_{2} \mathrm{O}_{2} \mathrm{Merck}$ : 100 Aq.) gehalten, hierauf mit physiol. Kochsalzlösung gewaschen und in den Paraffinofen (bis $60^{\circ}$ ) gebracht.

Beide werden braun, das mit $\mathrm{H}_{2} \mathrm{O}_{2}$ vorbehandelte dünkler braun als das mit $\mathrm{HCl}$. Die Stücke kommen abermals, u. zw. für 30 Stunden in Salzsäure resp. Wasserstoffsuperoxyd, wo sie, namentlich das Salzsäurestück sich beträehtlich aufhellen. Hierauf an der Luft liegend, wo sie trocknen, dunkeln sie wieder stark nach; diesmal ist das in $\mathrm{HCl}$ aufbewahrte Stück schwarzbraun geworden, vollständig dem Kontrollstück im Ofen gleichend, das in $\mathrm{H}_{3} \mathrm{O}_{2}$ aufbewahrte gelbbräunlich. 
Ähnlich verlief folgender Versuch, doch zeigte er, daß durch zu starke Reagentien die postmortale Färbungsfähigkeit leidet:

Haut eines jungen Mädchens (fung. gen.) wird mit etwas physiol. Kochsalzlösung bei $55^{\circ}$ gehalten (Kontrollstück auf Eis). Nach 24 Stunden tiefgraubraun geworden, wird es vom subkutanen Fett möglichst befreit, in 4 Teile geteilt, von denen $a$ in Kochsalzlösung (phys.), $b$ in Ameisensäure, $c$ in Salzsäure $(1: 10), d$ in $4 \%$ Wasserstoffsuperoxyd kommt.

$a$ wird etwas blässer, doch bleibt die Basalschicht deutlich dunkel;

$b$ wird bedeutend blässer;

$c$ wird bedeutend blässer, auch die Basalschicht viel heller;

$d$ wird entfärbt, so daß es ganz normal aussieht.

Alle Stücke werden halbiert und je eine Hälfte wird nach Auswaschen in physiol. Kochsalelösung - nur $d$ (Wasserstoffsuperoxyd) war nicht gewaschen worden - wieder in die Wärme gegebeu.

$a$ (Kochsalz) wird wieder dunkel,

$b$ (Ameisensäure) wird wieder dunkel, stark geschrumpft,

c) (Salzsäure) wird wieder dunkel, stark geschrumpft,

$d$ (Wasserstoffsuperoxyd) wird gelb (kadmiumgelb).

Eine Wiederkehr der dunklen Farbe durch Erwärmung war also in diesem Versuche wegen zu hoher Konzentration des zur Entfärbung verwendeten Wasserstoffsuperoxyds nicht mehr zu beobachten.

III.

a) Auch andere (physiologisch pigmentärmere) Gewebe bilden bei Erwärmung Pigment, aber nicht so auffallend, wie die Haut.

Frei präpariertes Fettgewebe wurde bei $58^{\circ} \mathrm{C}$ kadmiumgelb, Muskeln wurden lehmartig bräunlichgelb und nur dort dunkelbraun, wo sie dem heißen Glasgefäße direkt anlagen, also geröstet wurden.

b) Durch eingreifende chemische Operationen sind von der Haut aber auch von anderen Organen und Geweben pigmentierte Produkte (Huminsubstanzen) erhältlich.

Hievon habe ich mich (vor langer Zeit) bei der (behufs quantitativer Arsen- und Quecksilberbestimmung vorgenommenen) Zerstörung der Haut durch Salzsäure und Kaliumchlorat, sowie bei der im zugeschmolzenen Rohre ausgeführten Hydrolyse des Horns überzeugt. Mehrstündiges Kochen von Haut und Hautpulver in $12 \%$ iger Salzeäure auf dem Wasserbade gibt gefärbte Endprodukte: graubräunliche Flüssigkeit mit schwarzen Flöckchen; auch mit Fettgewebe-Eiweißlösungen und Muskeln ist dasselbe zu erzielen. 
Ein Beitrag zu Versuchen über postmortale Pigmentbildung. 301

Diese Versuche lassen daher nur eine Farbstoffbildung erschließen, die mehr von der chemischen Operation als von dem bestimmten Gewebe, z. B. der Haut, ablängig ist. Charakteristischer für eine stärkere Pigmentierungsfähigkeit der Haut scheinen die von $\mathrm{Königste} \mathrm{in} \mathrm{erhobenen} \mathrm{Befunde} \mathrm{mit} \mathrm{schwachen}$ Kalilösungen zu sein.

Von den Versuchen mit 1\% iger Lösung von kohlensauerem Natron, die ich aufstellte, verlief der erste folgendermaßen:

Nach 19 Stunden ist die Epidermis der betreffenden Stücke deutlich braun; nach zwei weiteren Tagen ist die Epidermis in der ganzen Dicke braun, u. 2 w. stärker als die im Ofen bei $57^{\circ}$ gehaltene, aber auch die Flūssigkeit ist eine Spur gelb geworden.

In einem zweiten und dritten Versuch dagegen war diese Bräunung nicht sehr auffallend, doch waren Bedingungen vorhanden, welche nach den genannten Autoren der Pigmentbildung nicht günstig sind. Denn die Haut des 2. Versuches war schon 6 Tage alt und an den Rändern bereits welk; sie brännte sich kaum im kohlensauren Natron, nur die sich alsbald ablösende Hornschicht war eine Spur brännlich.

Und auch im 2. Versuche kam eine durch mehrere Tage (4) konservierte Haut von einem Mammakarzinom zum Versuch; auch hier bloB die Hornschichte unmerklich graugelb.

Diese Bräunung durch schwache Kalilauge hat somit Ähnlichkeit mit jener durch Erwärmung.

Mit $1 \%$ iger Salzsäure dagegen habe ich in 19 und 43 Stunden eine stärkere Verfärbung der Epidermis nicht erzielt, doch ist eine bräunliche (blutige) des Unterhautzellgewebes eingetreten, welche durch die Epidermis durchschimmerte.

Kochen in Alkohol (Königstein) bewirkte bei einer schon von Natur pigmentierten Haut - Präputium - unter starker Schrumpfung tiefschwarz graue Pigmentierung, wobei etwas gelblich brauner Niederschlag im Alkohol sich absetzte.

c) Die folgenden Versuche beschäftigten sich mit der Möglichkeit, daß ein Ferment in den Epidermiszellen Pigment bilde. Sind doch Oxydasen, welche während des Lebens oder nach dem Tode auftretende Abbauprodukte z. B. Tyrosin in Pigment überführen, bei Pflanzen, niederen Tieren, in melanotischen Geschwiilsten von Säugetieren nachgewiesen worden. ${ }^{1}$ )

1) S. Literatur in O. v. F ürth: Phys. u. chem. Unters. ü. melan. Pig. Ztrbl. allg. P. u. path. A. 1904, XV, 15, daselbst ref. Landolt, 
Daß eine böhere Temperatur (bis $60^{\circ}$ ) die oxydative Tätigkeit der Gewebe nicht störe und demnach die postmortale Pigmentierung durch Wärme ganz wohl erklärt werden konnte, hat schon M eirowsky betont, aber auch für die im Leben statfindende, zu Zeiten gesteigerte Pigmentierung der Haut erscheint sie naheliegend, da mehrere mit Pigmentierung einhergehende Reize, wie Wärme, Belichtung, Röntgen-Bestrahlung Einfluß auf fermentative Prozesse haben, letztere hemmend oder steigernd. Auch das Vorhandensein von Stoffen, welche durch Wärme und Licht pigmentierbar sind, wie Tyrosin, Adrenalin u. a., in der Haut erscheint mit Rücksicht auf den Morbus Addison naheliegend; eine Anreicherung dieses Stoffes (oder einer Vorstufe desselben) im Organismus resp. in der Haut könnte die Pigmentvermehrung der letzteren während dieser Krankheit sehr wohl erklären.

Leider sind die nachfolgenden Versuche noch nicht imstande, die eine oder die zweite Voraussetzung für die Fermenthypothese sicherzustellen.

Ich versuchte durch Einwirkung von Tyrosin-, Hydrochinon-, Adrenalinlösungen eine innigere Haftung dieser Stoffe an den epidermoidalen Gebilden nachzuweisen, indem ich möglichst frisch von Lebenden und Leichen exstirpierte Haut in $1 \%$ ige schwach alkalische Lösungen dieser Stoffe brachte und sie bei Zimmer-, Brut- und Paraffinofentemperatur und in der Sonne durch Tage beließ. Von diesen Versuchen ergaben wohl namentlich die in der Wärme gehaltenen Proben, viel schwächer jene in der Sonne, eine insbesondere die Epidermis treffende Färbnng.

Namentlich schienen die bei $37^{\circ}$ gehaltenen Stücke dafür zu sprechen, indem ein deutlicher Unterschied der Epidermis und des Bindegewebes makroskopisch zu beobachten war. Viel langsamer trat eine Färbung im strahlenden Sonnenlichte (in Uviolröhren) und noch langsamer nach vielen Tagen bei Zimmertemperatur zu Tage. Die im Paraffinofen gehaltenen Stücke gaben dagegen sehr rasch tiefbraune, rotbraune oder schwarze Färbung der ganzen Hautstücke.

Die im Brutofen bei $37^{\circ}$ entstandene Gelbbraunfärbung der Haut ging bei längerem Auswaschen der Stücke in physiologischer Kochsalzlösung wieder zurück, so daß ein Unterschied der Tyrosin- und der in physiologischer Kochsalzlösung gehaltenen Stücke mikroskopisch nicht zu beobachten war. Die bei höherer Temperatur in den genannten Lösungen gehaltenen

Bertrand, Biedermann, v. Fürth u. Schneider, H. Pribram, Gessard. 
Ein Beitrag zu Versuchen über postmortale Pigmentbildung. 303

Stücke verloren ebenfalls in physiologischer Kochsalzlösung einen beträchtlichen Teil ihrer Färbung. Die epidermoidalen Teile behielten sie jedoch in ansehnlicher Stärke. Gefrierschnitte möglichst frischer Haut (soweit ich letztere von der chirurgischen Klinik erhalten konnte) in 1\%iger Hydrochinonlösung färbten sich im Paraffinofen und boten im mikroskopischen Bilde eine deutlich stärkere Färbung der Epidermis mit Akzentuierung der basalen Zellschicht und jener über dem Stratum granulosum sowie der epithelialen und endothelialen Hautgebilde. $\mathrm{Ob}$ jedoch diese und andere Erfahrungen für eine elektive, vielleicht durch oxydative Wirkung herbeigeführte Färbbarkeit der Epithelien sprechen und sich für die Lehre von der Pigmentbildung in diesem Sinne verwerten lassen, kann ich nicht sagen. Denn Lösungen von Tyrosin, Adrenalin, Hydrochinon nahmen schon bei gewöhnlicher Temperatur, namentlich aber im Brut- und Paraffinofen eine gelbliche bis dunkelbraune Färbung an, was für ihre schon an sich, namentlich aber in Gegenwart von organischen Stoffen stattfindende Oxydierbarkeit spricht und handelt es sich daher bei der betreffenden Färbung der Hautstïcke wahrscheinlich bloß um Adsorption und nicht um echte chemische Färbung. Doch soll auf diese merkwürdigen Dinge in einer späteren Mitteilung nochmals eingegangen werden.

Auch einen in der Haut durch Oxydation färbbaren Stoff habe ich bisher nicht sicher auffinden können.

An der Haut von Kaninchen, denen eine oder beide Nebennieren exstirpiert worden waren und die diesen Eingriff durch mehrere Stunden und Tage überstanden hatten, glaubte ich eine auffallende Schwärzung im Paraffinofen bemerkt zu haben. Ich habe daraufhin die (vor dem Tode kurz geschorene) Haut von normalen Kaninchen mit der hinreichenden Menge von physiologischer Kochsalzlösung (mit etwas Toluol) extrahiert und die abgepreßte und filtrierte Flüssigkeit auf Adrenalin untersucht. Als Reaktionen benützte ich die Vulpiansche ') Grünfärbung von verdünnter Eisenchloridlösung und die Ehrmannsche ${ }^{2}$ ) Pupillenerweiterung bei Temporarien.

1) S. Meth. d. Adrenalinnachw. bei A. Fränkel. A. f. exp. Path. u. $\mathrm{Ph} .60$, p. 395. In einem meiner Fälle gab der enteiweißte Kochsalzextrakt der Kaninchenhaut die Reaktion v. Fränkel-Allers-Krauss, Gelb- resp. Rotfärbung bei Erhitzen mit Kal. bijod u. Phosphorsäure und Ausschüttelung mit Chloroform.

2) Ehrmann: A. f. exp. P. u. Ph. B. 53 u. 55. 
In einigen Versuchen war der Erfolg ein positiver, in dem sich sowohl die Eisenchloridlösung bei Zusatz des Hautextraktes grün färbte, als in Pupillenversuchen deutlich und lang dauernde Pupillenerweiterung - verglichen mit der entsprechenden Kontrollpupille - einstellte; doch waren ebenso viele Versuche negativ.

Fasse ich das Resultat vorangehender Versuche zusammen, so ergibt sich:

Durch Erwärmung ist eine stärkere Färbung der Haut erzielbar (Meyrowsky, Königstein).

Der Grad derselben erscheint durch die hiebei stets auftretende Schrumpfung bedeutend erhöht.

Eintrocknen der Haut bewirkt eine Dunkelfärbung derselben, welche durch nachheriges Auf$q u \in l l e n$ wieder beseitigt wird.

Behandlung der Haut mit oxydierenden und reduzierenden Reagentien von nicht bedeutender Konzentration hindert das Eintreten der postmortalen Verfärbung nicht.

In der Haut des Kaninchens ist manchmal ein Stoff vorhanden, welcher mit dem Adrenalin durch die positive Eisenchloridreaktion (Grünfärbung) und die

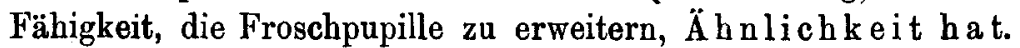

